\title{
Influence of contextual task constraints on preferred stride parameters and their variabilities during human walking
}

\author{
Lauro V. Ojeda ${ }^{1}$, John R. Rebula ${ }^{1,2}$, Arthur D. Kuo ${ }^{1}$, and Peter G. Adamczyk ${ }^{2, *}$ \\ ${ }^{1}$ Department of Mechanical Engineering, The University of Michigan, Ann Arbor, Michigan, USA \\ ${ }^{2}$ Intelligent Prosthetic Systems, LLC, Ann Arbor, Michigan, USA; *p.g.adamczyk@gmail.com
}

\begin{abstract}
Walking is not always a free and unencumbered task. Everyday activities such as walking in pairs, in groups, or on structured walkways can limit the acceptable gait patterns, leading to motor behavior that differs from that observed in more self-selected gait. Such different contexts may lead to gait performance different than observed in typical laboratory experiments, for example, during treadmill walking. We sought to systematically measure the impact of such task constraints by comparing gait parameters and their variability during walking in different conditions over-ground, and on a treadmill. We reconstructed foot motion from foot-mounted inertial sensors, and characterized forward, lateral and angular foot placement while subjects walked over-ground in a straight hallway and on a treadmill. Over-ground walking was performed in three variations: with no constraints (self-selected, SS); while deliberately varying walking speed (self-varied, SV); and while following a toy pace car programmed to vary speed (externally-varied, EV). We expected that these conditions would exhibit a statistically similar relationship between stride length and speed, and between stride length and stride period. We also expected treadmill walking (TM) would differ in two ways: first, that variability in stride length and stride period would conform to a constant-speed constraint opposite in slope from the normal relationship; and second, that stride length would decrease, leading to combinations of stride length and speed not observed in over-ground conditions. Results showed that all over-ground conditions used similar stride length-speed relationships, and that variability in treadmill walking conformed to a constant-speed constraint line, as expected. Decreased stride length was observed in both TM and EV conditions, suggesting adaptations due to heightened awareness or to prepare for unexpected changes or problems. We also evaluated stride variability in constrained and unconstrained tasks. We observed that in treadmill walking, lateral variability decreased while forward variability increased, and the normallyobserved correlation between wider foot placement and external foot rotation was eliminated. Preferred stride parameters and their variability appear significantly influenced by the context and constraints of the walking task.
\end{abstract}

Keywords: gait variability; treadmill walking; task constraint; goal-equivalence; wearable sensor; inertial sensor 
37 Human walking can entail multiple goals such as regulation of speed, avoidance of excess energy expenditure, and maintenance of balance. In terms of gait parameters such as average stride length, frequency, and width, there are generally multiple combinations that can satisfy a single goal such as walking speed [1-3]. And for a given speed, it has long been observed that the preferred stride length coincides with minimum metabolic energy expenditure [4-6]. There are, however, a variety of contexts that may determine a person's walking speed. For example, speed may be governed intrinsically for a person walking solo, or extrinsically when one matches the speed of another person or group. The goal of matching speed could potentially conflict with or override the normal gait preference, on average or from stride to stride. We therefore seek to determine whether and how the context for walking speed may shape a person's gait preferences.

47 The preferred stride length normally increases with greater walking speed. This may be described through a nonlinear relationship [7,8], in which stride length ? (defined between two same-side footfalls) increases with ? ? ? ? simpler linear approximation proportional to speed [8]. Either relationship also determines stride

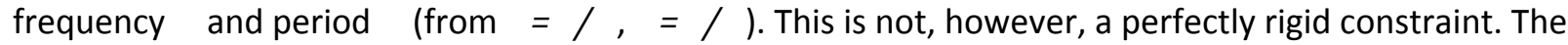
preferred relationship is different when humans walk to match a given stride length or frequency [9]. This behavior is consistent with minimization of energetic cost (which varies as a function of gait parameters), subject to a constraint. A broader view is therefore that gait parameters depend on what variables are constrained, and therefore the context of the walking task.

There are many possible contexts for walking. Stride frequency may occasionally be constrained, for example if one walks to the beat of music. Stride length may be constrained for walking over stepping stones or to avoid obstacles, and both length and frequency may be constrained when marching in formation. Alternatively, walking speed may be determined externally, for example when one person wishes to match the speed of another. Less natural, but common in the laboratory, is for speed to be controlled by a treadmill or by the time between photo-gates overground. These contexts may be subtly different, because each case provides different cues and allowances regarding speed. The speed of a group of people can potentially vary, and so any single individual may be tracking a changing speed, perhaps with some subjective allowance for momentarily deviating from the group. If that allowance is small, the strategy for tracking a changing speed may favor relatively short and brief strides to facilitate faster speed corrections. A treadmill usually imposes fixed reference speed, and its length imposes limits on allowable deviations in the person's speed, both of which may affect the preferred stride parameters. And if a treadmill's speed is not fixed, that may also result in altered stride parameters [10]. The context that is perhaps most difficult to define is normal walking, which may entail an intrinsic goal such as reaching a particular destination within a desired time frame. Depending on such a goal, the minimization of energy expenditure generally results in different sets of optimal gait parameters [3]. It is unknown whether or how an imposed speed constraint, such as those used in laboratory experiments, will differ from unconstrained walking in situ. 
A Over-ground

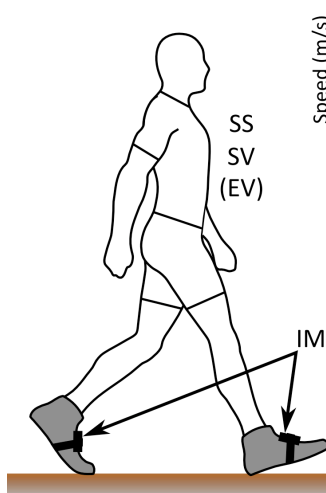

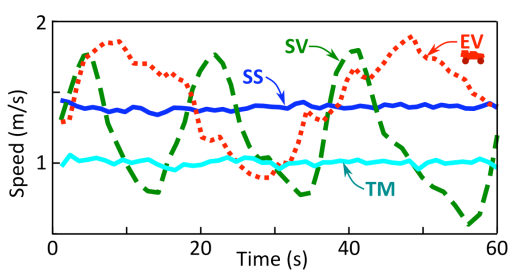

B Treadmill

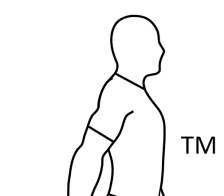

M

.
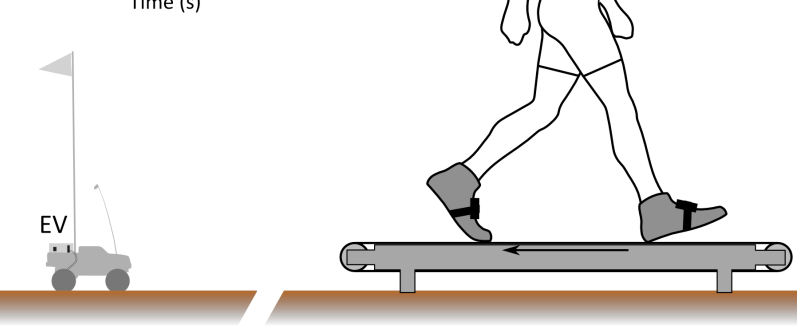

An additional feature of gait parameters is their variation relative to mean. Each stride parameter exhibits variability depending in part on the average stride parameters and walking speed (e.g., Danion et al. 2003; Sekiya et al. 1997). Some variabilities are also correlated, with manifestations such as covariance between stride length and width [13], stride length and speed [14], or stride width and foot angle [15]. Here, context also plays an important role, because a treadmill constrains allowable variations, resulting in a particular stride covariance pattern that limits fluctuations in speed due to the treadmill's restricted length $[16,17]$. Overground walking is almost certainly different, especially when there is no explicit speed reference. In fact, humans exhibit relatively larger fluctuations in speed than in stride length, width, or frequency, suggesting that intrinsic speed regulation may be quite relaxed compared to the desire to optimize stride length [14]. However, lacking a specific comparison of stride covariances under different contexts such as treadmill vs. overground, it remains to be proven whether stride variations are also context dependent.

The purpose of the present study was to compare how contexts such as self-selecting speed vs. tracking a reference speed, or walking over ground vs. on a treadmill, affect human gait parameters and their variability. We directly compared stride parameters and their variability among free and constrained over-ground walking tasks and treadmill walking using the same instrumentation and computational techniques. We hypothesized that tracking tasks might cause small shifts in the preferred stride relationship toward shorter strides, to accommodate stride corrections made for tracking. We also expected that a treadmill's constant-speed constraint would reduce variability in stride length and speed, compared to free over-ground walking. Furthermore, those fluctuations should co-vary according to the speed constraint, with fluctuations violating the constraint resulting in relatively fast corrective responses. We tested for such effects to explore how even subtle differences in the context of walking can affect parameters that are normally considered indicative of biomechanical or neural control function.

\section{Methods} We measured gait parameters of healthy adult human subjects walking in free and speed-controlled conditions over-ground, and at constant speed on a treadmill. We estimated forward, lateral and angular foot placement and stride period using inertial sensors worn on each foot. We computed 
metrics to describe these parameters and their variabilities as a function of contexts such as selfselected speed, an external reference requiring tracking of speed, or treadmill-fixed speed. A total of 23 subjects participated, 12 Young ( $22.4 \pm 4.2$ years (mean \pm S.D.), 4 male, 8 female; leg length $0.91 \pm 0.06$ $\mathrm{m}$ ) and 11 Elderly ( $65.7 \pm 3.0$ years (one not reported), 5 male, 6 female; leg length $0.89 \pm 0.07 \mathrm{~m}$ ), treated as a single group for analysis. Over-ground conditions were tested in an indoor hallway, approximately $112 \mathrm{~m}$ long in a straight line and $2.4 \mathrm{~m}$ wide, with a smooth tile floor and fluorescent lighting. The treadmill was a split-belt instrumented research treadmill (Bertec Corp., Columbus, Ohio, USA; $1.83 \mathrm{~m}$ long, $0.83 \mathrm{~m}$ wide) with computer-controlled speed, and a centerline gap of about $20 \mathrm{~mm}$. Subjects wore their customary walking shoes, to which inertial sensors (APDM Inc., Portland, OR, USA) were mounted on the upper forefoot outside the shoe using elastic polyurethane tape (Fig. 1). The sensors sampled three-axis angular velocity (range $2000 \mathrm{deg}^{-1}$ ) and linear acceleration (range $6 \mathrm{~g}^{\prime} \mathrm{s}$ ) continuously at $128 \mathrm{~Hz}$. Subjects gave their written informed consent according to University of Michigan Institutional Review Board policies.

Four contextual variations of walking were performed (Fig. 1). One was over-ground at self-selected speed (SS). In a second condition, termed self-varied (SV), subjects were asked to intentionally vary their speed on their own. The third condition, termed externally varied (EV), asked subjects to follow a remote-controlled toy car and track its changing speed. Finally, one trial was performed on the treadmill (TM) at a fixed speed, chosen by the subject for comfort. The self-selected speed averaged $1.38 \mathrm{~m} \cdot \mathrm{s}-1$ (average S.D. of speed within a trial: $0.05 \mathrm{~m} \cdot \mathrm{s}-1$ ), whereas the treadmill speed averaged $1.02 \mathrm{~m} \cdot \mathrm{s}-1$ (average S.D. $0.03 \mathrm{~m} \cdot \mathrm{s}-1$ ). Self-varied speeds fluctuated between roughly 0.8 and $1.9 \mathrm{~m} \cdot \mathrm{s}-1$, and externally-varied speeds fluctuated between roughly 0.6 and $1.85 \mathrm{~m} \cdot \mathrm{s}-1$. The speed of the car in the externally-varied condition (EV) was controlled by programming motor duty cycle to follow a pseudorandom sum-of-sines pattern with 4 frequency components $(0.1,0.05,0.03$ and $0.02 \mathrm{~Hz})$. The one treadmill condition was level, constant speed walking for 1-3 minutes according to the speed and endurance capability determined for each subject through trial-and-error.

Stride Trajectory Reconstruction Because traditional motion capture cannot be used for extended distances over-ground, we estimated stride parameters from inertial data, collected from synchronized inertial measurement units mounted on the two feet [18]. Stride distances and periods were obtained by numerically integrating angular 


\section{A Reconstructed Stride Trajectory}

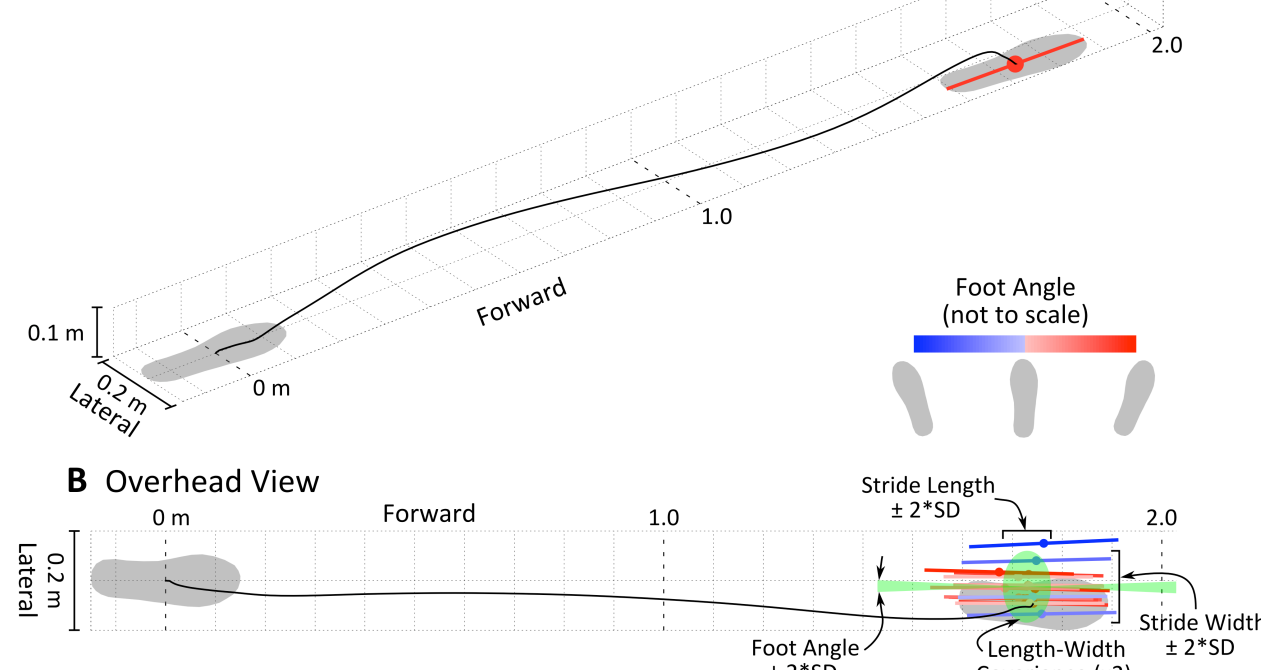

C Side View

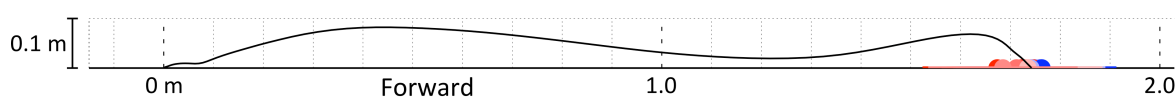

Figure 3: Stride trajectory reconstruction and foot placement statistics. (A) Example stride, reconstructed from acceleration and angular velocity signals from a footmounted IMU. (B) Top view of the same stride, with 16 surrounding footfalls overlaid to show variability (dots, color coded with lines for angle). Variability in forward, lateral, and angular foot placement are shown by linear and angular brackets. Covariation between forward and lateral foot placement is shown with a covariance ellipse. Variability limits shown are at 2 standard deviations from the mean. (C) Side view of the same stride, with other foot placements overlaid (dots).

velocity and linear acceleration data to provide angular and Cartesian position of the foot (Figs. 2-3), using custom drift correction methods based on zero foot velocity during each stance phase, as described previously $[18,19]$. Foot position and orientation were sampled at each footfall. To eliminate initiation and termination of gait, the beginning and end of each trial were discarded using a speed threshold of $80 \%$ of the trial mean speed.

\section{Stride Parameters Measured and Calculated}

We defined forward, lateral, and angular foot placement as the position of the foot sensor at each footfall relative to the local forward direction. The local forward for each footfall was defined as the best-fit line in a centered moving window of seven footfalls (see Fig. 2A). Forward foot placement (equivalently, stride length [?) was the forward displacement from the previous footfall (Fig. 2B). Lateral foot placement (stride width ?) was the orthogonal distance to the right of the local forward line (Fig. 2B). Angular foot placement (foot angle ?) was defined as the heading about a vertical axis: the clockwise planar rotation angle of the foot relative to the forward line, as viewed from above (Fig. 2B; this measure has an arbitrary offset). Stride period was determined by threshold crossings, ending when horizontal-plane foot velocity first exceeded $0.2 \mathrm{~m} \mathrm{~s}^{-1}$ following each footfall (shortly before toe-off; Fig. 2C). Stride speed was computed as stride length divided by stride period. Individual strides were removed from the analysis if there was an obvious discrete gait deviation, such as a sudden turn to avoid an obstacle; these events were verified by video to ensure all undisturbed strides were kept. All metrics were computed for both left and right feet, so that each stride produced two data points - one for each foot. The number of strides analyzed per trial was $140 \pm 15$ (mean \pm SD) over ground and $179 \pm$ 90 on the treadmill (a wide range due to subjects' different capabilities). 
Data from the self-varied speed condition

155

156

157

158

159

160

161

162

163

164

165

166

167

168

169

170

171

172

173

174

175

176

177

178

179

180

181

182

183

184

185

186

187

188

189

190

191

192

(SV) were used to determine preferred relationship [7],

$$
\text { ? }=\text { ? ? } * \text { ? 目 }
$$

The self-varied speed (SV) condition was we consider it a relatively natural range of speeds.

We compared each subject's gait parameters from the self-selected (SS), externally-varied (EV) and treadmill (TM) conditions against the self-varied (SV) reference to determine whether stride length was shorter during speed-tracking stride parameters for over-ground walking as a function of speed. We computed a best fit to each subject's stride length vs. stride velocity data using an established nonlinear used as the basis for comparison, because representation of normal behavior across a tasks. We computed the mean speed in the SS, EV and TM conditions, and the mean stride length in SS and TM conditions. For EV condition, a second curve of the same form (Eq. 1) was computed, and evaluated at the mean speed to give an observed stride length. At each condition's mean speed, we evaluated the subject-specific SV curve fit to determine the normal stride length, and subtracted this from the observed stride length, yielding a stride length deviation: the amount by which the observed stride length differed from the normal stride length (Fig. 4A). We compared this stride length deviation (which was typically negative, i.e. strides shorter than expected) across conditions using ANOVA and a Holm-Sidak step-down procedure (Fig. 4B).

We also compared the variability of foot placement measurements between over-ground (SS) and treadmill (TM) walking, in terms of standard deviation and covariance (Figs. 3, 5). We compared the standard deviation of forward, lateral, and angular foot placement across conditions using ANOVA. Forward placement was detrended with respect to stride velocity prior to computing its standard deviation, according to each subject's stride length vs. stride speed relationship from the SV condition [14]. We evaluated the covariance of lateral vs. forward, lateral vs. angular, and forward vs. angular foot placement. We compared the covariance from SS and TM conditions using ANOVA .

We further evaluated whether any of the controlled conditions altered the internal structure of gait variability. For each subject and condition, we computed the best-fit line to stride length vs. stride period data (圆 vs. ?) using a total-least-squares technique (Fig. 6A). The slope was expected to be negative in all over-ground conditions, as suggested by the normal preferred relationship [7], but positive in treadmill walking, compatible with the constant-speed constraint [17]. 
Finally, we further analyzed fluctuations in self-selected overground (SS) and treadmill (TM) conditions by decomposing fluctuations with respect to the mean into components along and orthogonal to a line of constant speed. We evaluated the standard deviation of each component to examine the quantity of variability in each direction, and performed detrended fluctuation analysis (DFA) on each component to determine persistence [17]. Standard deviations and DFA exponents were compared using ANOVA (Fig. 7).

\section{Results}

We found that subjects walked with largely similar preferred stride relationships across all over-ground conditions (Fig. 4). As expected, there was a smaller range in speed in the selfselected (SS) speed condition than in the self-varied (SV) condition, but they both appeared to obey the same preferred stride relationship. However, the externally-varied (EV) speed condition resulted in
A
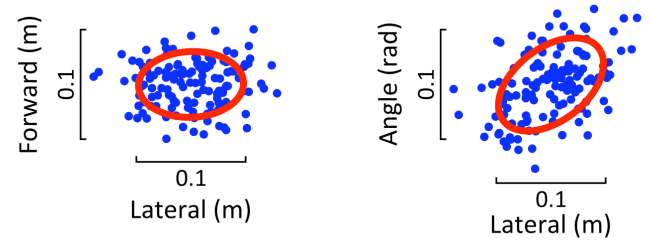

B

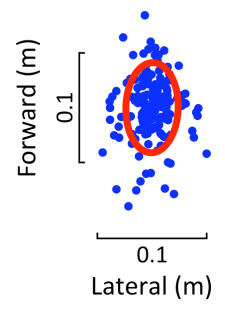

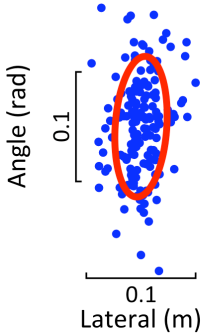

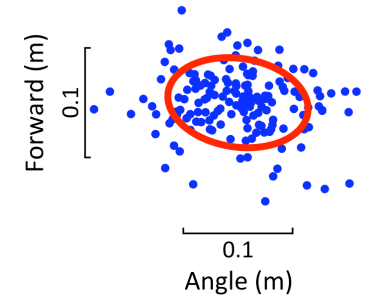

C Foot Placement - Standard Deviation

D Foot Placement - Covariance

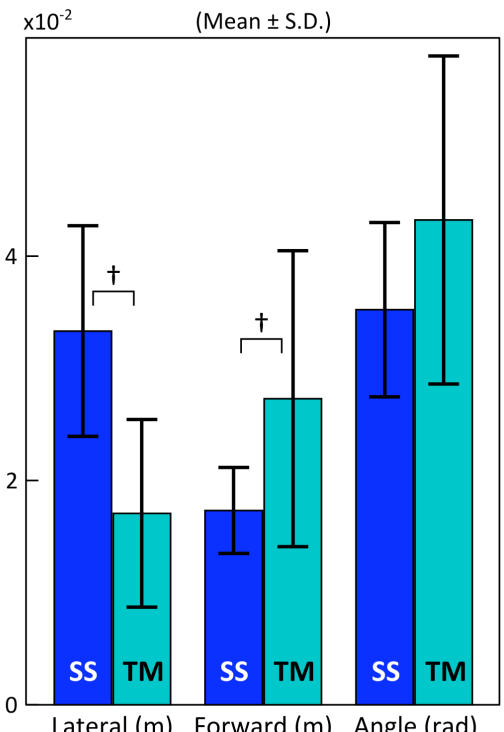

Lateral (m) Forward (m) Angle (rad)

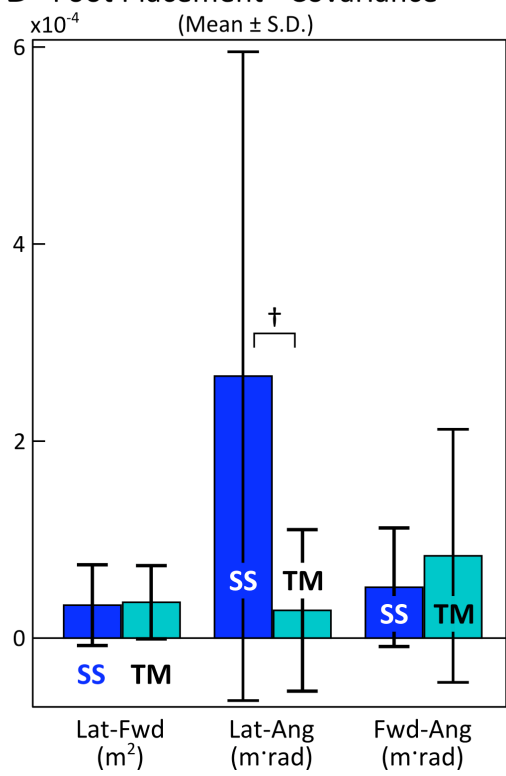

Figure 5: Overall foot placement variability during (A) self-selected (SS) and (B) treadmill (TM) walking. (C) Standard deviation of foot placement decreased in the lateral direction, increased in the forward direction, and remained constant in the angular direction. (D) Covariance between lateral and angular foot placement was eliminated during treadmill walking. + Significant difference, TM vs SS, $P<0.005$. restructuring of variability to lie along the constant-speed constraint, as well as a decrease in persistence of fluctuations violating this constraint. Treadmill walking also changed the relative magnitude of variability in stride length vs. stride width, and eliminated the covariation between lateral and angular foot placement observed overground. These results are presented in detail below.

The preferred stride length decreased in the two conditions of external speed reference, EV and TM (Fig. 4). Compared to the self-varying (SV) condition, strides were about $9 \%$ shorter in the treadmill (TM) condition (stride length deviation $-0.109 \pm 0.116 \mathrm{~m}, P=2 \mathrm{e}-4$ ), and $3 \%$ shorter in the externally-varied 
(EV) condition (stride length deviation -0.041 $\pm 0.045 \mathrm{~m}, P=3 \mathrm{e}-4)$. In contrast, stride lengths during self-selected (SS) walking were not different from selfvaried (SV) walking ($0.006 \pm 0.043 \mathrm{~m}, P=$ 0.49).

Overall gait variability was different when walking on a treadmill versus over ground (Fig. 5). On the treadmill, lateral variability was reduced $(P=6 \mathrm{e}-6)$ and forward foot placement variability was increased $(P=0.002)$

(Fig. 5A,C). Angular foot placement variability tended to increase slightly in treadmill walking (Fig. 5C), though the effect did not reach adjusted significance $(P=0.012)$. Covariation (Fig. 5B,D) between the different measures of foot placement was also altered on the treadmill. The covariances of forward vs. lateral foot placement and of forward vs. angular foot placement were small in both over-ground and treadmill walking (all positive, $P<0.005$ ), and were not different between SS and TM conditions $(P>$ 0.26). However, the covariance of lateral vs. angular foot placement was significantly positive in overground walking $(P=8 \mathrm{e}-4)[15]$, whereas it was statistically zero in treadmill walking $(P=0.11)$. This reduced covariance is partially attributable to the reduced variance of lateral foot placement.

In relation to the speed constraint, covariation between stride length and stride period was structured differently in the treadmill condition, compared to the overground condition (Fig. 6). The best-fit slope of stride length vs. stride period (Fig. 6B) was negligible (modest value but with extreme variability) in SS walking $(P=0.8)$ and significantly negative in SV and EV walking $(P<0.001)$, but significantly positive in the TM condition $(P<0.001)$. The extreme variability in slope in SS walking was due to noise dominating the best-fit procedure in the absence of correlation between stride length and stride period.

Variability magnitude during SS was roughly equal in directions aligned with a line of constant-speed (tangential; S.D. $0.026 \pm 0.005$, arbitrary units) and orthogonal to this line (S.D. $0.033 \pm 0.008$ ) (Fig. 7). In $\mathrm{TM}$, variations were about twice as great $(P=4 \mathrm{e}-6)$ tangential to the constant-speed line (S.D. $0.042 \pm$ 0.021 ), as orthogonal to it (S.D. $0.022 \pm 0.009$ ). The increase in variability tangential to the constantspeed line in TM compared to SS was statistically significant $(P=0.002)$, as was the decrease in 
274 Persistence of fluctuations in forward foot

275 placement and stride period appeared to be

276 reduced during TM walking in comparison to SS walking. The DFA exponent ? describes the growth of error in a linear process model with reducing amounts of local detrending, to indicate persistence (19>0.5) or anti-persistence ( $>0.5$ ) of fluctuations. This exponent was not different in SS vs. TM for fluctuations tangential to the constantspeed line $(0.78 \pm 0.15$ for SS vs. $0.81 \pm 0.20$ for $\mathrm{TM} ; P=0.49)$, but was significantly different for fluctuations orthogonal to it $(0.91 \pm 0.18$ for SS vs.

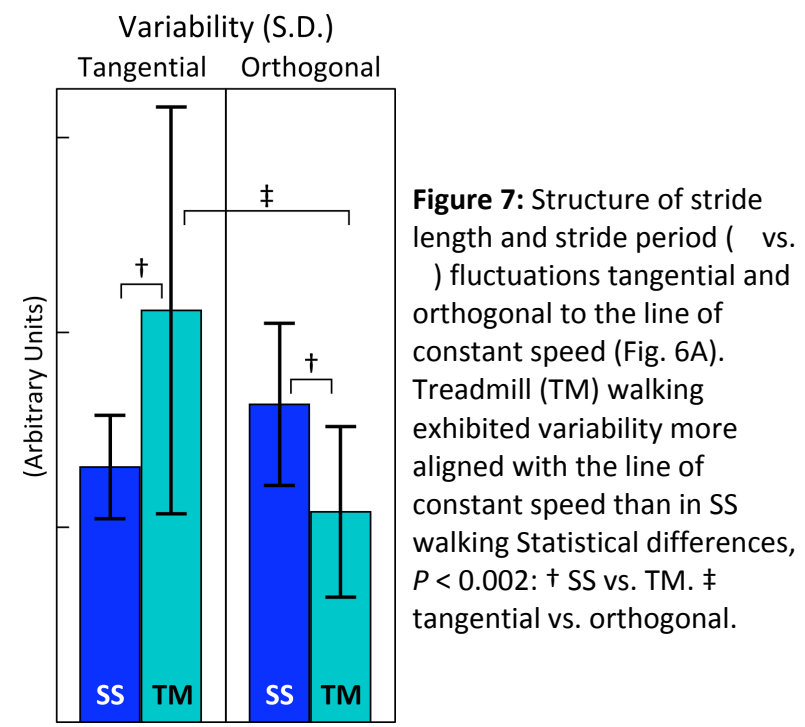
$0.46 \pm 0.11$ for TM; $P<1 \mathrm{e}-6)$. The lower DFA exponent in TM suggests that fluctuations that change speed decay more rapidly in treadmill than in over-ground walking.

\section{Discussion}

Prior research has shown that stride length and stride period variability on a treadmill are related in a structured way that is approximately compatible with the constant-speed constraint, described as a goal-equivalent manifold $[16,17]$. Our findings extend this result by directly comparing the structure of variability in the same individuals walking "normally" (over-ground in a straight line) and on a treadmill. For the same individuals, variability is structured more along a constant-speed constraint during treadmill walking than during hallway walking, even though the hallway walking was also at constant speed. It appears that the presence of a constant speed constraint, rather than the act of actually walking at constant speed, is the factor that changes the structure of gait variability.

The structure of spatial foot placement variability (forward, lateral, and angular) was perturbed in treadmill walking in comparison to over-ground walking. On the treadmill, lateral variability decreased while forward variability increased (Fig. 5A). Lateral variability on the treadmill was also less than forward variability, a finding opposite to other observations [20-22]. We suspect that our results were caused by the visual cue provided by the mid-line gap in the split-belt treadmill used for the test, as well as the treadmill's relatively narrow width compared to a hallway. This is another contextual factor that may be associated with differences between treadmills, independent of the difference from overground walking. A more common feature of most treadmills is the constant-speed constraint. In the forward direction, this constraint, or perhaps the penalty for violating it (falling off the treadmill), could represent a secondary attention task that interferes with the walking task, leading to increased variability overall [23]. Differences in self-motion may also play a role in the difference between treadmill and over-ground walking. Perceived lateral motion could be enhanced by the absence of interference from rearward optic flow, as lateral aspects of optic flow are known to influence lateral steering [24-26]. Or, lack of forward optic flow on a treadmill may reduce the information available to regulate speed, again leading to increased variability $[25,27,28]$. Such effects could be investigated 
separately by experimentally varying optic flow in a virtual reality environment with an automated

313 position-maintaining treadmill.

314 The observed organization of stride length vs. stride period variability along the constant-speed line was 315 expected in treadmill walking, according to principles of control in the presence of motor redundancy $316[16,29]$. However, it was surprising that this was achieved as much by increased variability along the constant-speed constraint as by reduced variability orthogonal to it (Fig. 7). The increase in forward foot placement variability likely contributed to this effect. However, for this outcome measure, the secondary task of regulating position also reduces variability orthogonal to the constant-speed line. Future experiments regulating other aspects of gait (e.g. stride period with a metronome, stride length with stepping stones) may show whether controlling variability in a particular outcome generally increases orthogonal variability, or whether it is possible to reduce variability in some tasks without such 323 a penalty.

324 In addition to direct reduction of orthogonal variability, compliance with the constant-speed constraint may be implemented by rejecting speed disturbances more quickly on a treadmill. Temporal organization of variability such that speed fluctuations are quickly, rather than gradually, rejected could allow short-term average speed to remain within a narrow range even with larger stride-to-stride deviations. The reduced DFA exponent for fluctuations orthogonal to the constant-speed constraint (i.e., speed-varying fluctuations) supports this idea. It may be that both strategies - direct speed variability reduction and quick rejection of speed changes - are used in treadmill walking. Implementing either strategy adds a task to the treadmill context that is not present in normal over-ground walking, with potential consequences for motor control.

Treadmill walking also disturbed the normally-observed correlation between wider foot placement and external foot rotation (see Figs 3, 5), in which "wider" steps are also slightly more "toe-out" [15]. This small but consistent effect was eliminated on the treadmill, and the reduction in lateral-angular correlation appeared greater than would be explained by the modest reduction in lateral variability alone. It may be that the treadmill's constant direction of travel disallows minor steering corrections normally used in a hallway. Or, the absence of rearward optic flow may allow improved discernment of both lateral and angular motion, allowing better control. In either case, it appears that the usual correlation between lateral deviation and external rotation is a behavioral choice, perhaps to benefit balance [15], and not a physiological coupling.

One surprising finding was the extent to which strides were shorter than expected in both of the speedcontrolled tasks (externally-varied EV and treadmill TM, Fig. 4). Shorter than normal stride length has been reported in some [30-35], but not all [36], literature on treadmill effects. We found that strides were roughly $9 \%$ shorter on the treadmill (TM) speed than at the same speed in the self-varied (SV) speed condition. Additionally, we found that even in a hallway, the act of following a pace car (EV) led to reduced stride length, by roughly $3 \%$. These adaptations were not required by the tasks. We speculate that subjects may adopt a shorter stride as a simple consequence of heightened attention, to increase their safety margin in conditions that feel risky (TM), or to prepare for unexpected speed changes (EV) [37-39]. 
This study shows multiple differences between treadmill walking and walking over ground, predominantly in how gait variability is structured. Future studies of variability, including steady-state variability and cycle-to-cycle variations due to discrete events, should carefully consider whether these contextual effects will bias the results. The constraints of treadmill walking may structure a person's adaptations in a way that eliminates some of their preferred responses to perturbations. Thus, experiments using perturbations to determine human behavior could be distorted if performed in a highly constrained context, such as a treadmill that requires constant speed and prevents changes in direction.

These effects of treadmill walking could not be observed previously, because traditional measurement methods have limited capture volumes or walkway lengths that prevent extended bouts of steady walking. Other techniques using wearable sensors can capture bouts of extended duration, but none to date enable reconstruction of three-dimensional foot position and orientation without a previously calibrated model of gait. Using foot-mounted inertial sensors with strapdown motion reconstruction enabled a direct comparison between extended walks over-ground and on a treadmill, using the same sensors and algorithms for both data sets. In future work, movement in unconstrained spaces such as homes, streets, public buildings and hiking trails can be studied with this approach.

One limitation in this study is that some subjects walked slower on the treadmill than in the selfselected over-ground condition, because they were uncomfortable on the treadmill. This speed mismatch could bias direct comparisons of these conditions, such as the standard deviation and covariance of foot placement measures. Given that we observed both increases and decreases in different components of variability, it seems unlikely that this minor speed mismatch is responsible for all the results. Nevertheless, further direct comparison could be made at matched speeds to verify the results.

\section{Conclusions}

Studies of variability in biomechanical task performance can provide useful insight into motor control. Measuring variability to identify motor strategies requires many cyclic task repetitions, a requirement that generally requires a simulated environment in a laboratory. However, the simulated environment can limit the solutions available to the motor system, resulting in changes to the structure of motion variability that can confound experimental results. The potential for such disturbances must be taken into account in the design of variability studies. When possible, mobile measurement techniques may be preferable to prevent such experimental artifacts. Foot-mounted inertial sensors provide one such system, which can be useful for studies of gait.

\section{Acknowledgments}

This work was supported in part by the U.S. National Institute of Aging (R44AG030815), the U.S. Department of Defense (W81XWH-09-2-0142), and U.S. Office of Naval Research (ETOWL). The content is the responsibility of the authors and does not necessarily represent the views of any agency. Work was approved by the University of Michigan Institutional Review Board (HUM00020554). The authors thank K. Alex Shorter and Daniel Kowalsky for their assistance in data collection and subject recruitment. 
390 Dr. Adamczyk is a member of Intelligent Prosthetic Systems, LLC, which develops systems for analyzing 391 gait using techniques related to those described in this project.

\section{References}

393

394

395

[1] Donelan JM, Kram R, Kuo AD. Mechanical work for step-to-step transitions is a major determinant of the metabolic cost of human walking. J Exp Biol 2002;205:3717-27.

[2] Donelan JM, Kram R, Kuo AD. Mechanical and metabolic determinants of the preferred step width in human walking. Proc R Soc Lond B 2001;268:1985-92. doi:10.1098/rspb.2001.1761.

[3] Long LL, Srinivasan M. Walking, running, and resting under time, distance, and average speed constraints: optimality of walk-run-rest mixtures. J R Soc Interface 2013;10:20120980. doi:10.1098/rsif.2012.0980.

[4] Elftman H. Biomechanics of Muscle. J Bone Jt Surg 1966;48-A:363-77.

[5] Ralston HJ. Energy-speed relation and optimal speed during level walking. Int Z Für Angew Physiol Einschließlich Arbeitsphysiologie 1958;17:277-83. doi:10.1007/BF00698754.

[6] Zarrugh MY, Todd FN, Ralston HJ. Optimization of energy expenditure during level walking. Eur J Appl Physiol 1974;33:293-306. doi:10.1007/BF00430237.

[7] Grieve DW. Gait patterns and the speed of walking. Biomed Eng 1968;3:119-22.

[8] Grieve DW, Gear RJ. The Relationships Between Length of Stride, Step Frequency, Time of Swing and Speed of Walking for Children and Adults. Ergonomics 1966;9:379-99. doi:10.1080/00140136608964399.

[9] Bertram JE, Ruina A. Multiple walking speed-frequency relations are predicted by constrained optimization. J Theor Biol 2001;209:445-53. doi:10.1006/jtbi.2001.2279.

[10] Snaterse M, Ton R, Kuo AD, Donelan JM. Distinct fast and slow processes contribute to the selection of preferred step frequency during human walking. J Appl Physiol 2011;110:1682-90. doi:10.1152/japplphysiol.00536.2010.

[11] Danion F, Varraine E, Bonnard M, Pailhous J. Stride variability in human gait: the effect of stride frequency and stride length. Gait Posture 2003;18:69-77. doi:10.1016/S0966-6362(03)00030-4.

[12] Sekiya N, Nagasaki H, Ito H, Furuna T. Optimal walking in terms of variability in step length. J Orthop Sports Phys Ther 1997;26:266-72.

[13] Bauby CE, Kuo AD. Active control of lateral balance in human walking. J Biomech 2000;33:1433-40.

[14] Collins SH, Kuo AD. Two Independent Contributions to Step Variability during Over-Ground Human Walking. PLOS ONE 2013;8:e73597. doi:10.1371/journal.pone.0073597.

[15] Rebula JR, Ojeda LV, Adamczyk PG, Kuo AD. The Stabilizing Properties of Foot Yaw in Human Walking. J Biomech in review.

[16] Cusumano JP, Dingwell JB. Movement variability near goal equivalent manifolds: Fluctuations, control, and model-based analysis. Hum Mov Sci 2013;32:899-923. doi:10.1016/j.humov.2013.07.019.

[17] Dingwell JB, John J, Cusumano JP. Do Humans Optimally Exploit Redundancy to Control Step Variability in Walking? PLoS Comput Biol 2010;6:e1000856. doi:10.1371/journal.pcbi.1000856.

[18] Rebula JR, Ojeda LV, Adamczyk PG, Kuo AD. Measurement of foot placement and its variability with inertial sensors. Gait Posture 2013;38:974-80. doi:10.1016/j.gaitpost.2013.05.012.

[19] Ojeda L, Borenstein J. Non-GPS navigation for security personnel and first responders. J Navig 2007;60:391. 
[20] Owings TM, Grabiner MD. Step width variability, but not step length variability or step time variability, discriminates gait of healthy young and older adults during treadmill locomotion. J Biomech 2004;37:935-8. doi:10.1016/j.jbiomech.2003.11.012.

[21] Owings TM, Grabiner MD. Variability of step kinematics in young and older adults. Gait Posture 2004;20:26-9. doi:10.1016/S0966-6362(03)00088-2.

[22] Dean JC, Alexander NB, Kuo AD. The effect of lateral stabilization on walking in young and old adults. leee Trans Biomed Eng 2007;54:1919-26. doi:10.1109/TBME.2007.901031.

[23] Yogev-Seligmann G, Rotem-Galili Y, Mirelman A, Dickstein R, Giladi N, Hausdorff JM. How Does Explicit Prioritization Alter Walking During Dual-Task Performance? Effects of Age and Sex on Gait Speed and Variability. Phys Ther 2010;90:177-86. doi:10.2522/ptj.20090043.

[24] Warren WH, Kay BA, Zosh WD, Duchon AP, Sahuc S. Optic flow is used to control human walking. Nat Neurosci 2001;4:213-6. doi:10.1038/84054.

[25] Lamontagne A, Keshner EA, Bugnariu N, Fung J. Virtual Reality Reveals Mechanisms of Balance and Locomotor Impairments. In: Weiss PL (Tamar), Keshner EA, Levin MF, editors. Virtual Real. Phys. Mot. Rehabil., Springer New York; 2014, p. 169-202.

[26] Sarre G, Berard J, Fung J, Lamontagne A. Steering behaviour can be modulated by different optic flows during walking. Neurosci Lett 2008;436:96-101. doi:10.1016/j.neulet.2008.02.049.

[27] Schubert M, Prokop T, Brocke F, Berger W. Visual kinesthesia and locomotion in Parkinson's disease. Mov Disord Off J Mov Disord Soc 2005;20:141-50. doi:10.1002/mds.20281.

[28] Konczak J. Effects of Optic Flow on the Kinematics of Human Gait: A Comparison of Young and Older Adults. J Mot Behav 1994;26:225-36. doi:10.1080/00222895.1994.9941678.

[29] Scholz JP, Schöner G. Use of the Uncontrolled Manifold (UCM) Approach to Understand Motor Variability, Motor Equivalence, and Self-motion. In: Levin MF, editor. Prog. Mot. Control, Springer New York; 2014, p. 91-100.

[30] Watt JR, Franz JR, Jackson K, Dicharry J, Riley PO, Kerrigan DC. A three-dimensional kinematic and kinetic comparison of overground and treadmill walking in healthy elderly subjects. Clin Biomech 2010;25:444-9. doi:10.1016/j.clinbiomech.2009.09.002.

[31] Wass E, Taylor NF, Matsas A. Familiarisation to treadmill walking in unimpaired older people. Gait Posture 2005;21:72-9. doi:10.1016/j.gaitpost.2004.01.003.

[32] Zeni Jr. JA, Higginson JS. Gait parameters and stride-to-stride variability during familiarization to walking on a split-belt treadmill. Clin Biomech 2010;25:383-6. doi:10.1016/j.clinbiomech.2009.11.002.

[33] Alton F, Baldey L, Caplan S, Morrissey MC. A kinematic comparison of overground and treadmill walking. Clin Biomech 1998;13:434-40. doi:10.1016/S0268-0033(98)00012-6.

[34] Strathy GM, Chao EY, Laughman RK. Changes in knee function associated with treadmill ambulation. J Biomech 1983;16:521-2. doi:10.1016/0021-9290(83)90066-0.

[35] Riley PO, Paolini G, Della Croce U, Paylo KW, Kerrigan DC. A kinematic and kinetic comparison of overground and treadmill walking in healthy subjects. Gait Posture 2007;26:17-24. doi:10.1016/j.gaitpost.2006.07.003.

[36] Matsas A, Taylor N, McBurney H. Knee joint kinematics from familiarised treadmill walking can be generalised to overground walking in young unimpaired subjects. Gait Posture 2000;11:46-53. doi:10.1016/S0966-6362(99)00048-X.

[37] Hak L, Houdijk H, Steenbrink F, Mert A, van der Wurff P, Beek PJ, et al. Stepping strategies for regulating gait adaptability and stability. J Biomech 2013;46:905-11. doi:10.1016/j.jbiomech.2012.12.017.

[38] Hak L, Houdijk H, Steenbrink F, Mert A, van der Wurff P, Beek PJ, et al. Speeding up or slowing down?: Gait adaptations to preserve gait stability in response to balance perturbations. Gait Posture 2012;36:260-4. doi:10.1016/j.gaitpost.2012.03.005. 
480

481

482

483
[39] Hak L, Houdijk H, Beek PJ, van Dieën JH. Steps to Take to Enhance Gait Stability: The Effect of Stride Frequency, Stride Length, and Walking Speed on Local Dynamic Stability and Margins of Stability. PLoS ONE 2013;8:e82842. doi:10.1371/journal.pone.0082842. 